\title{
Epidural Anaesthesia: How Easy Is It to Walk on Quicksand?
}

\author{
Constantin Bodolea*
}

University of Medicine and Pharmacy "Iuliu Hatieganu“ Cluj Napoca, Romania

The effectiveness of neuraxial blockade remains a very debatable issue. Many orthopaedic surgical procedures can be performed using either a single spinal shot, an epidural catheter neuraxial blockade, or general anesthesia.

Memtsoudis (2013) reviewed nearly 400.000 patients undergoing primary hip or knee arthroplasties compared neuraxial versus general anesthesia, and reported that the 30-day mortality, the length of stay, the hospital cost and the in-hospital complications were all was significantly lower than with other forms of anesthesia [1]. Similarly Helwan (2015) in a study comparing general with, regional anesthesia for total hip arthroplasty reported a reduction in deep surgical site infection rates, the length of hospital stay, postoperative cardiovascular rates, and pulmonary complications [2]. However, a recent systematic review of more than 10.000 patients enrolled in randomized control trials and prospective comparative studies, found no statistically significant differences between spinal or epidural blockade and general anaesthesia with respect to mortality, surgical duration, surgical site of infections, nerve palsies, postoperative nausea and vomiting or thromboembolic diseases, when thrombo-prophylaxis was used. The authors concluded that there is limited evidence to support the view that neuraxial anesthesia is superior to general anesthesia with regards to postoperative outcomes [3].

Although the incidence of complications following epidural placement is low, these are distressing and challenging for anesthetist, carrying as they do, a significant risk of litigation.

Because epidural catheter insertion is based on anatomical landmarks, many factors can influence its misplacement. An audit carried out by the Royal College of Anaesthetists investigated all the relevant complications of central neural blocks (CNBs) performed over one year. Only two episodes of epidural catheter migration were reported out of a total of 293,050 performed epidurals. There were no reports of serious complications due to misplacement of an epidural catheter [4].

The most common causes of epidural analgesia failure are the intravascular, subdural, intrathecal and subcutaneous catheter placement. Reports also exist describing the misplacements of epidural catheters in the proximity of inferior vena cava, aorta, pleural cavity and the lumbar plexus [5-7].

Hermanides (2012) was of the opinion that epidural failure is more common than typically recognized in clinical practice, and identified technical factors, such as catheter misplacement, inappropriate choice of drug and dosage as potential failure factors [8].

Ruzman (2014) investigating 316 patients who underwent different surgical procedures using CNBs, tried to identify patient dependent factors such as age, gender, height, weight, body mass index, and quality of anatomical landmarks, technique dependent factors, such as the type of block, needle gauge, and patient positioning, and provider dependent factors such as the level of experience, as principal reasons associated with problematic neuraxial block. The authors found that "first puncture success" was related to younger age, lower weight and body mass index, while spine deformity, poor identification of interspinous space, a recumbent position during the puncture and the use of a paramedian approach, were associated with "first puncture failure" [9].

An observational study of 253 patients, of whom 131 received subarachnoid block and 122 an epidural block, reported that the provider's level of experience and the distance from skin to subarachnoid or epidural space influenced the difficulty in performing a neuraxial block [10]. Body mass index was considered to be a weak predictor of neuraxial block difficulty, in agreement with the earlier report by Chien (2003) [11].

The skillfulness of anaesthesiologist is an important factor in the overall successful rate in performing the 
neuraxial block. In a previous study, concerning young anesthetists at varying levels of learning neuraxial block, Kopacz (1996) concluded that 20-25 procedures each were necessary before an improvement in spinal and epidural anesthesia techniques was seen, and if a $90 \%$ success rate was set, 45 and 60 attempts at spinal and epidural anesthesia, respectively, may be necessary to achieve a reported improvement [12].

As regards epidural anaesthesia using the loss of resistance technique (LOR), this was first described by Dogliotti eighty-three years ago. He used fluid as the medium for injection as the needle passes through the various tissue structures on its path towards the epidural space.[13] Over time, the LOR technique has undergne various changes, with some authorities preferring to use air instead of fluid in locating the epidural space. Today, the two most commonly used media are air and saline.

In a retrospective analysis, Saberski reviewed the complications associated with the use air in LOR, noting complications such as pneumocephalus, retroperitoneal air, subcutaneous emphysema, venous air embolism, a greater incidence of incomplete analgesia and a higher rate of paresthesias consecutive to spinal cord and nerve root compression in conjunction with the simultaneous administration of nitrous oxide and positive pressure ventilation. He also reported that there was a propensity for less experienced practitioners to use a larger volume, particularly when technical difficulties were encountered when confirming placement of the epidural needle [14]. His advice was to limit the amount of air used, as much as possible, as the volume that can be safely injected into the epidural space has not been established. It is obvious that repeated injections of large quantities of air are more likely to be associated with complications than a single small volume injection, and there are reports of pneumocephalus when as little as three $\mathrm{ml}$ has been used [15]. Similar conclusions are shared by Shenouda and Cunningham who emphasized the superiority of saline versus air in the epidural LOR technique [16].

In this issue of The Journal of Critical Care Medicine, Isakov et al. described a very rare complication following a difficult attempt at epidural anaesthesia using LOR with air in an 81-old man undergoing hip surgery [17]. Following an injection of about $35 \mathrm{ml}$ of air, on the fourth postoperative day, the patient complained of moderate abdominal pain without peritoneal irritation signs. Subsequent computed tomography examination identified an accumulation of air in the retroperitoneal space, around the left psoas muscle with slight extension toward the left paravertebral muscle mass. The clinical manifestation and the general clinical context raised different questions regarding the differential diagnostic of abdominal pain.

Returning to the issue of an epidural anesthetic block, the possibility of failure is a reality that must not cause despair in the anesthetist's mind. However, according to the good clinical practice, patients must also be informed of the possibility of failure.

Anesthetists have need of a full and comprehensive understanding of the theoretical and practical issues regarding epidural anesthetic techniques, with the added requirement of having a rescue plan in cases of failure.

With this professional knowledge, the unwanted and the most dangerous complications related to epidural anesthesia will be avoided.

\section{CONFLICT OF INTEREST}

Nothing to declare.

\section{DEFERENCES:}

1. Memtsoudis SG, Sun X, Chiu YL, et al. Perioperative comparative effectiveness of anesthetic technique in orthopedic patients. Anesthesiology. 2013;118:1046-58. doi: 10.1097/ ALN.0b013e318286061d.

2. Helwan M, Avidan MS, Abdallah AB, et al. Effects of regional versus general anesthesia on outcomes after total hip arthroplasty. J Bone Joint Surg Am. 2015;97:186-93. doi: 10.2106/JBJS.N.00612.

3. Johnson RL, Kopp SL, Burkle CM et al. Neuraxial vs general anaesthesia for total hip and total knee arthroplasty: a systematic review of comparative effectiveness research. $\mathrm{Br} \mathrm{J}$ Anaesth. 2016;116:163-76. doi: 10.1093/bja/aev455.

4. Cook TM, Counsell DJ, Wildsmith AW. Major complications of central neuraxial block: report on the third national audit project of the Royal College of Anaesthetists. Br J Anaesth. 2009;102:179-90. doi: 10.1093/bja/aen360.

5. Amir A, Lash V, Moore A et al. Management of a rare complication of epidural analgesia in an obese laterally positioned parturient catheter entrapment next to the inferior vena cava. Reg Anesth Pain Med. 2015;40:726-8. doi: 10.1097/ AAP.0000000000000324.

6. Koch J, Nielsen JU. Rare misplacements of epidural catheters. Anesthesiology. 1986;65:556-7.

7. Inoue S, Nishimine N, Furuya H. Unintentional intrapleural insertion of an epidural catheter: should we remove it or leave it in situ to provide perioperative analgesia? Anesth Analg. 
Available online at: www.jccm.ro

2005;100:266-8. DOI: 10.1213/01.ANE.0000140241.97973.73.

8. Hermanides J, Hollmann MW, Stevens MF, Lirk P. Failed epidural: causes and management. Br J Anaesth. 2012;109:144-54. doi: 10.1093/bja/aes214.

9. Ruzman T, Gulam D, Harsanji I et al. Factors associated with difficult neuraxial blockade. Local and Reg Anesth. 2014:74752. doi: 10.2147/LRA.S68451.

10. Kim JH, Song SY, Kim BJ. Predicting the difficulty in performing a neuraxial blockade. Korean J Anesthesiol. 2011;61:377-81. doi: 10.4097/kjae.2011.61.5.377.

11. Chien I, Lu IC, Wang FY, et al. Spinal process landmark as a predicting factor for difficult epidural block: a prospective study in Taiwanese patients. Kaohsiung J Med Sci. 2003;19:563-8. DOI: 10.1016/S1607-551X(09)70507-8.

12. Kopacz DJ, Neal JM, Pollock JE. The regional anesthesia "learning curve". What is the minimum number of epidural and spinal blocks to reach consistency? Reg Anesth. 1996;21:182-90.
The Journal of Critical Care Medicine 2016;2(4) • 155

13. Dogliotti AM. A new method of block anesthesia. Segmental peridural spinal anesthesia. Am J Surg. 1933;20:107-18. doi:10.1016/S0002-9610(33)91041-7.

14. Saberski LR, Kondamuri S, Osinubi OY. Identification of the epidural space: Is loss of resistance to air a safe technique?A review of the complications related to the use of air. Reg Anesth Pain Med. 1997;22:3-9.

15. Vasder GM, Chantigian RC. Pneumocephalus following the treatment of a postdural puncture headache with an epidural saline infusion. J Clin Anesth. 1994;6:508-11. doi:10.1016/09528180(94)90094-9

16. Shenuda PE, Cunningham BJ. Assessing the superiority of saline versus air for use in the epidural loss of resistance technique: a literature review. Reg Anesth Pain Med. 2003;28:48-53. DOI: 10.1053/rapm.2003.50009

17. Isakov A, Shtein A, Kyzer S. Pneumoperitoneum after attempted epidural anesthesia. J Crit Care Med. 2016;2:198-200. DOI: 10.1515/jccm-2016-0029. 